\title{
A randomized trial comparing the clinical efficacy and safety of a novel steerable percutaneous kyphoplasty with traditional PKP in osteoporotic vertebral fractures
}

\author{
Jizheng Li ${ }^{1 \#}$, Xiaofeng Yuan ${ }^{2 \#}$, Fanbing Li ${ }^{1}$, Yi Ding ${ }^{3}$, Gang Ma ${ }^{4}$, Chao Song ${ }^{5}$, Xuesong Chen ${ }^{5}$ Enbin Wang ${ }^{6}$, \\ Jiaping Cui ${ }^{7}$, Qingli Kong ${ }^{8}$, Youqing Huang', En Song ${ }^{10}$
}

${ }^{1}$ Department of Orthopedics, First Clinical Medical College of Yunnan University of Traditional Chinese Medicine, Kunming, China; ${ }^{2}$ Department of Orthopedics, First People's Hospital of Kunming, Kunming, China; ${ }^{3}$ Department of Orthopedics, Guizhou Provincial People's Hospital, Guiyang, China; ${ }^{4}$ Department of Orthopedics, Southern Central Hospital of Yunnan Province, Mengzi, China; ${ }^{5}$ Pain Department, Yunnan Kungang Hospital, Kunming, China; ${ }^{6}$ Department of Orthopedics, Baoshan Hospital of Traditional Chinese Medicine, Baoshan, China; ${ }^{7}$ Department of Orthopedics, First People's Hospital of Zhaotong, Zhaotong, China; ${ }^{8}$ Department of Orthopedics, People's Hospital of Chuxiong Yi Autonomous Prefecture, Chuxiong, China; ${ }^{9}$ Pain Department, Second Affiliated Hospital of Kunming Medical University, Kunming, China; ${ }^{10}$ Department of Sports Medicine, First Affiliated Hospital of Kunming Medical University, Kunming, China

Contributions: (I) Conception and design: E Song; (II) Administrative support: F Li, C Song, G Ma; (III) Provision of study materials or patients: Y Ding, E Wang, F Li; (IV) Collection and assembly of data: Q Kong, Y Huang, X Chen; (V) Data analysis and interpretation: E Song, J Li, J Cui; (VI) Manuscript writing: All authors; (VII) Final approval of manuscript: All authors.

"These authors contributed equally to this work.

Correspondence to: En Song. Department of Sports Medicine, First Affiliated Hospital of Kunming Medical University, No. 295 Xichang Road, Kunming 650031, China. Email: 641634233@qq.com.

Background: Percutaneous kyphoplasty (PKP) is a highly practical technology to treat osteoporotic vertebral compression fractures (OVCFs). However, the operation time and radiation exposure remain problematic. This study explored the differences in surgical effects and safety between a novel steerable percutaneous kyphoplasty (S-PKP) and traditional PKP in order to achieve better clinical outcomes for OVCF patients. It is also exploring whether the new technology could reduce the radiation exposure.

Methods: This study recruited 72 patients (between March 2019 and January 2020) with OVCFs (single vertebra). The patients were semi-randomly divided these patients into two groups according to ID numbers: a S-PKP group (33 cases) and a PKP group (39 cases). We evaluated the clinical efficacy using the kyphotic Cobb angle, Oswestry disability index (ODI), visual analogue scale (VAS) score, injected cement volume, operation time, intraoperative radiation times, bone cement leakage, and postoperative complications. Patients were followed up once preoperatively, and at 1 day, 6 months, and 1 year postoperatively.

Results: There were no cases of cement leakage or postoperative complications. There were no significant differences in gender, age, Bone mineral density T-score (BMD T) value, Cobb angle between the two groups $(\mathrm{P}>0.05)$. Intraoperative bone cement injection was approximately $5.25 \pm 1.37$ and $5.32 \pm 1.29 \mathrm{~mL}$ in the PKP and S-PKP groups respectively. The postoperative VAS score and ODI of the two groups at 1 day, 6 months, and 1 year were markedly lower than before $(\mathrm{P}<0.05)$. There was a considerable improvement in the Cobb angle postoperatively $(\mathrm{P}<0.05)$. However, as the follow-up time extended, the Cobb Angle increased. The operation time and X-ray exposure times of patients in the PKP group were notably higher than those in the S-PKP group. The operation time was $51.59 \pm 9.14 \mathrm{~min}$ in the PKP group and $30.76 \pm 4.82 \mathrm{~min}$ in the S-PKP group. The frequency of intraoperative radiation was $105.9 \pm 31.93$ times in the PKP group and $47.42 \pm 11.88$ times in the S-PKP group.

Conclusions: Early results showed that S-PKP is a safe and efficient method for the treatment of OVCFs. $\mathrm{S}-\mathrm{PKP}$ can reduce the operation time and radiation exposure.

Trial registration: Chinese Clinical Trial Registry ChiCTR2100046727 
Keywords: Osteoporosis; vertebral compression fracture (OVCF); steerable percutaneous kyphoplasty (S-PKP); percutaneous kyphoplasty (PKP)

Submitted Feb 26, 2021. Accepted for publication Jun 15, 2021.

doi: $10.21037 /$ atm-21-1880

View this article at: https://dx.doi.org/10.21037/atm-21-1880

\section{Introduction}

Osteoporosis a systemic disease caused by the decrease of bone density and bone mass. Trauma fractures of the vertebrae are one of the most common complications of osteoporosis, referred as osteoporotic vertebral compression fracture (OVCF) (1). In 1984, Galibert et al. (2) demonstrated for the first time that vertebroplasty could be achieved by injecting bone cement into the vertebral body under minimally invasive conditions, which significantly alleviated the pain caused by osteoporotic fractures and increased the stability of the fractures. However, the minimally invasive methods at that time were insufficient in the recovery efficiency of vertebral height and the control of intraspinal cement leakage.

In 1998, the percutaneous kyphoplasty (PKP) procedure improved the problems associated with percutaneous vertebroplasty (PVP) with respect to vertebral height recovery and bone cement leakage by using balloon dilation and progressive expansion of the compression space of the fractured vertebral body, as well as injection of polymethylmethacrylate (PMMA). Yan et al. (3) confirmed that both PVP and PKP surgery could effectively relieve pain in patients with OVCFs, restore vertebral height, and correct the Cobb angle. Therefore, PKP and PVP are safe and effective surgical methods for the treatment of OVCFs (4). However, compared with PVP, PKP surgery takes longer and the material is relatively expensive $(4,5)$. In terms of surgical procedures, bilateral puncture is considered to be a relatively safe and effective mainstay method $(6,7)$. Studies have shown that unilateral puncture can also achieve the effect of bilateral puncture, and the efficacy is comparable with bilateral puncture (8). In a short-term follow-up study assessed by radiographic outcome, bilateral PKP was found to be a better solution to restore the anterior vertebral height (9). Furthermore, a relatively new study has shown that unilateral PKP exhibits a higher risk of re-fracture of adjacent vertebral bodies compared with bilateral PKP (10).

Therefore, a surgical approach that accounts for the advantages of unilateral and bilateral PKP is needed to resolve the above controversy. We believe that novel steerable percutaneous kyphoplasty (S-PKP) technology is one of numerous potential solutions. This study aimed to evaluate the surgical effects and safety of the novel S-PKP compared with traditional PKP for the treatment of thoracolumbar OVCFs. We present the following article in accordance with the CONSORT reporting checklist (available at https://dx.doi.org/10.21037/atm-21-1880).

\section{Methods}

\section{Patient demographics}

In this study, a retrospective semi-randomized controlled trial was conducted. From March 2019 to January 2020, patients from multi-center hospitals in Yunnan and Guizhou were enrolled in this study. And it recruited 72 patients diagnosed with OVCFs (single vertebra) according to the Chinese Guidelines for the Diagnosis and Treatment of Primary Osteoporosis [2017]. The patients were semirandomly assigned to two groups based on whether the last digit of ID numbers (generated via a computerized. The even-number group, 33 patients received S-PKP (S-PKP group), the odd-numbered group, which included 39 patients, received traditional PKP (PKP group). The Data and Safety Monitoring Board, blinded to treatment determined group. In total, there were 11 males and 28 females in the PKP group, and 9 males and 24 females in the S-PKP group. The segments of the collapsed vertebrae in the two groups were as follows: thoracic vertebrae in 21 cases, including T10 in 6 cases, T11 in 10 cases, and T12 in 5 cases; lumbar vertebrae in 51 cases, including L1 in 9 cases, L2 in 16 cases, L3 in 15 cases, L4 in 8 cases, and L5 in 3 cases. In the PKP group, there were 12 cases of thoracic fracture and 27 cases of lumbar fracture. In the SPKP group, there were 9 cases of thoracic fracture and 24 cases of lumbar fracture (Figures 1,2). All of the ethical requirements for clinical trials in this study were approved by the Medical Ethics Committee of the First Affiliated Hospital of Yunnan University of Traditional Chinese Medicine (2021-034). All procedures involving human participants were performed in accordance with the Declaration of Helsinki (as revised in 

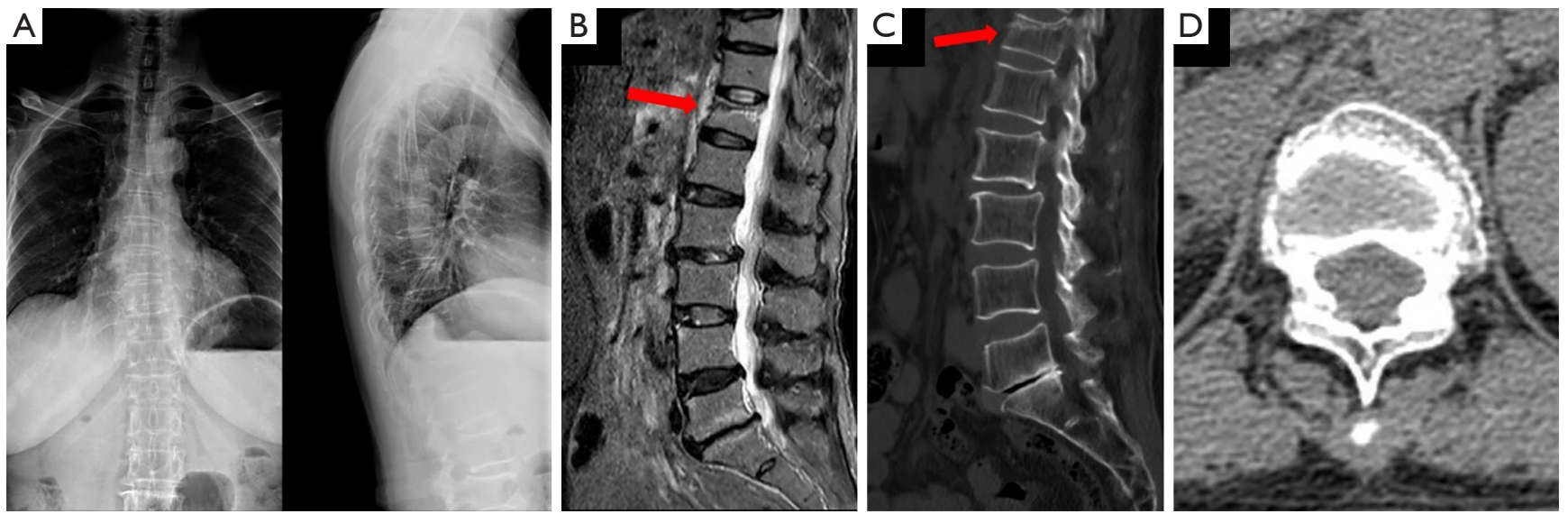

Figure 1 Case one: A 69-year-old female patient. (A) Preoperative thoracic radiological images revealing a compression fracture at the T12 level; (B) preoperative MRI images revealing a fresh compression fracture at the T12 level (arrow); (C,D) preoperative CT scan revealing compression fractures at the T12 level (arrow). CT, computed tomography; MRI, magnetic resonance imaging.
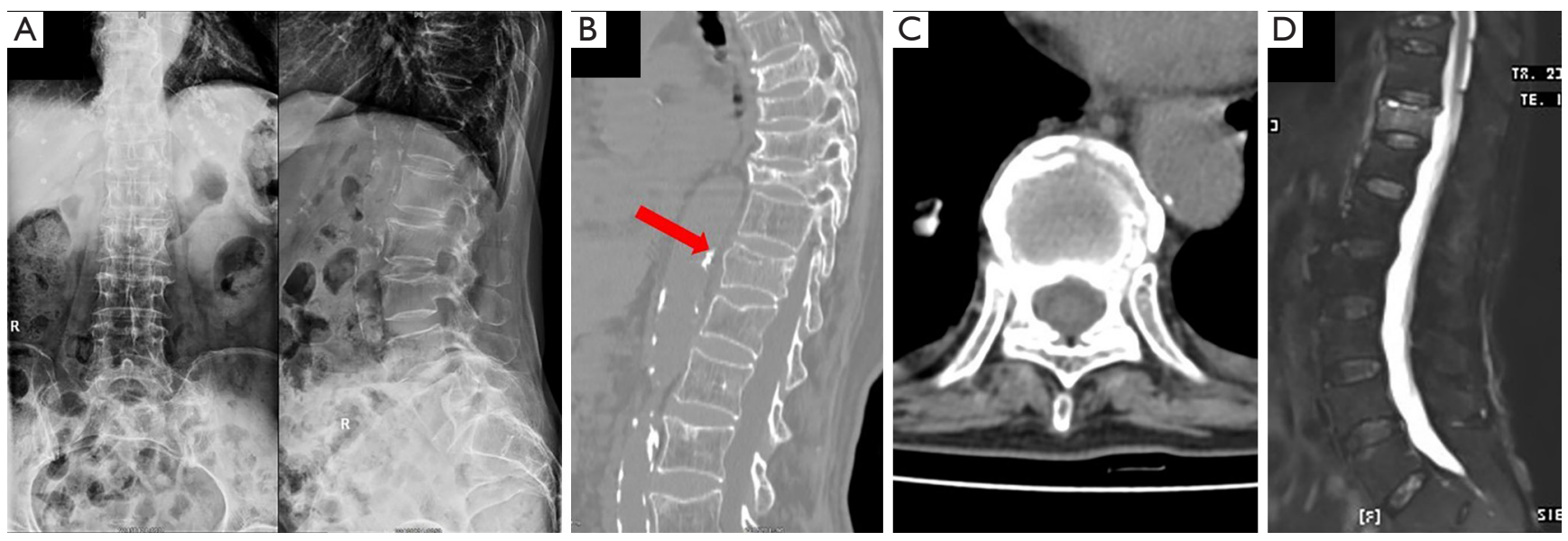

Figure 2 Case two: A 73-year-old female patient. (A) Preoperative lumbar and thoracic radiological images revealing a compression fracture at the T11 level; (B) a preoperative CT scan revealing compression fractures at the T6, T7, T8, T9, and T11 levels (arrow); (C) preoperative MRI images revealing a fresh compression fracture at the T11 level. CT, computed tomography; MRI, magnetic resonance imaging.

2013). Informed consent was obtained from all patients.

\section{Selection criteria}

The inclusion criteria for patients enrolled in this study were as follows: (I) patients diagnosed with fresh OVCF on $\mathrm{X}$-ray, computed tomography (CT), and magnetic resonance imaging (MRI), and also suffered from back pain; (II) patients with a single vertebral compression fracture from the 10th thoracic vertebra to the 5th lumbar vertebra; (III) bone mineral density (BMD) was measured by dual-energy $\mathrm{X}$-ray and was lower than -2.5 ; and (IV) patients received a follow-up observation at least 1 year after surgery.

The exclusion criteria were as follows: (I) patients that had previously undergone PKP or PVP surgery; (II) patients who could not cooperate with the operation due to various reasons, or those who requested conservative treatment; (III) patients who presented with pathological fractures other than osteoporosis; (IV) patients with nerve root or spinal cord compression symptoms; and (V) patients with coagulopathy.

If the patient died perioperatively, or the surgery failed, or the patient was unable to continue treatment after enrolment, special analysis was performed if it was relevant to the trial, or data exclusion was performed if it was not. 


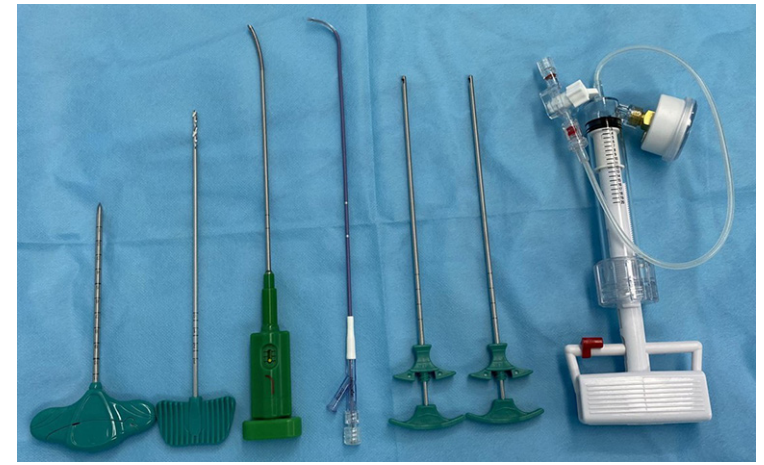

Figure 3 Steerable percutaneous kyphoplasty (S-PKP) instruments (from left to right): puncture needle, bone drill, steerable curved bone expander, curved balloon tamp, bone void filler device with side hole, and inflation syringe with volume scale.

In the PKP group, four participants were excluded. Two patients were lost to follow-up at 6 months postoperatively, and another two patients were lost to follow-up at 1 year postoperatively. In the S-PKP group, three participants were excluded. We were informed by a family member of one participant at the 6-month postoperative follow-up stage that they had suffered a hip fracture due to a second fall. Another two patients were lost to follow-up at 1 year postoperatively. The lost cases were treated as missing data in the statistical analysis.

\section{Materials}

The S-PKP and traditional PKP instruments used for the OVCFs were produced by Shanghai Legend Medical \& Technology Co., Ltd. (Shanghai, China) (Figure 3). The bone cement was produced by Synimed Synergie Ingénierie Médicale S.A.R.L. (Chamberet, France). The contrast agent, iohexol, was obtained from the Yangze River Pharmaceutical Group Co., Ltd. (Jiangsu, China).

\section{Surgical procedure}

\section{S-PKP group}

Patients who received topical anesthesia were placed in the prone position. The surgical procedure was monitored by fluoroscopy (Siemens C arm Xmedical equipment, Germany). A unilateral transpedicular approach was performed with puncture needle systems under biplanar fluoroscopic guidance. Next, the surgeon used lateral view fluoroscopy to determine that the bone drill had penetrated into the anterior $75 \%$ of the vertebral body (Figure 4). Subsequently, a steerable curved bone expander (Shanghai Legend Medical \& Technology Co., Ltd.) was inserted through the cannula. Three scale lines on the handle of the steerable curved bone expander indicate the different depths of insertion into the vertebral body. When the depth reached the first scale, the knob was turned at the end of the handle clockwise 180 degrees, at which point the expander head began to bend to the opposite side. When the depth reached the second scale, the knob was turned at the end of the handle clockwise 360 degrees, at which point the expander head could be bent to the midline of the vertebral body. When the depth reached the third scale, the knob was turned at the end of the handle clockwise 360 degrees, at which point the expander tip could be bent to the contralateral pedicle projection on the anteroposterior radiograph. When the ideal position was reached, the knob was turned at the end of the handle of the bone expander counter clockwise until the rotation stopped, and the bone expander could then be pulled out (Figures 5,6). Subsequently, the curved balloon tamp (Shanghai Legend Medical \& Technology Co., Ltd.) was inserted through the cannula, advanced into the vertebral body, and inflated in the middle area of the vertebral body by injecting the contrast agent, as determined by AP (anteroposterior) and lateral view fluoroscopy (Figures 7,8,9). Once the Cobb angle and vertebral height were obtained to the suitable condition, which had been determined by radiography intraoperatively, the surgeon stopped injecting the contrast agent and quitted the balloon. Where the balloon was withdrawn, the bone cement was injected into the collapsed vertebral body using a bone void filler device (Shanghai Legend Medical \& Technology Co., Ltd.) with a side hole (Figures 10,11,12). Patients were required to bed rest for the first 24 hours after surgery.

\section{PKP group}

Like the S-PKP procedure, patients who received the PKP operation were also placed in the prone position under local anesthesia. Moreover, the monitoring methods, operative route, and penetration depth were the same as the S-PKP procedure. However, there was no angle adjustment for the knob to bend to the contralateral pedicle projection in a more appropriate way. Consistent with S-PKP, the balloon was slowly inflated by injecting the contrast agent through a high-pressure pump (Shanghai Legend Medical \& Technology Co., Ltd). The opportunity to extract the contrast agent and withdraw the balloon, the dosage, and 

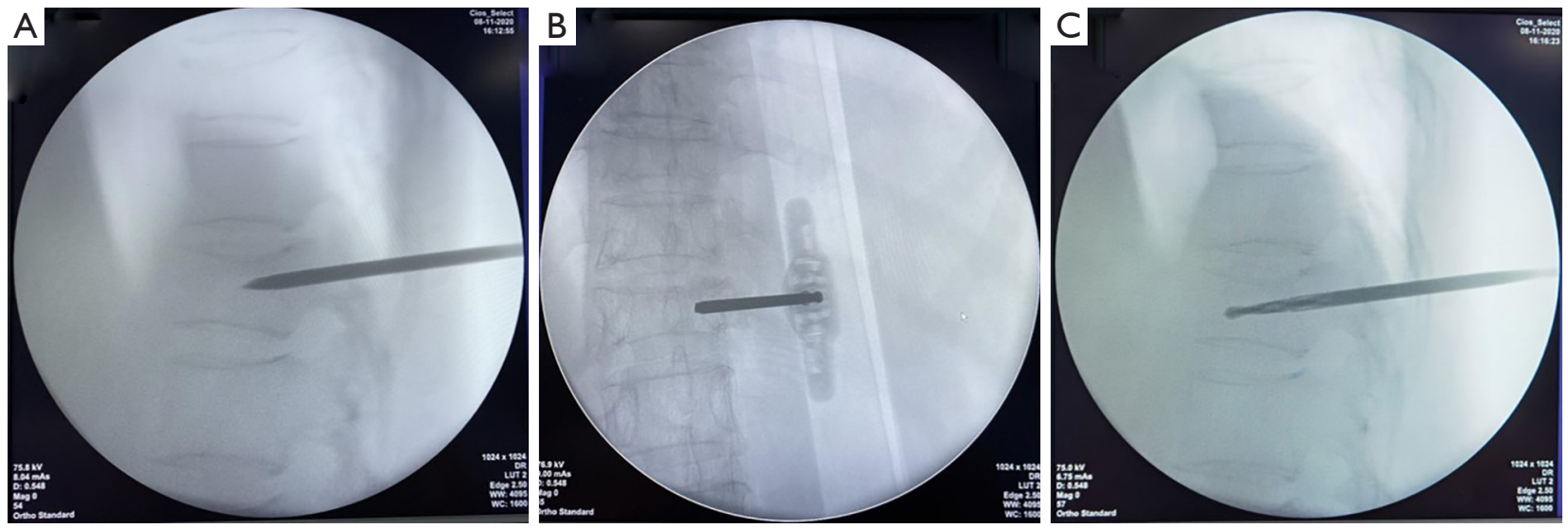

Figure 4 Steerable percutaneous kyphoplasty (S-PKP) surgical procedure in case one: $(\mathrm{A}, \mathrm{B})$ Unilateral transpedicular approach; $(\mathrm{C})$ bone drill advanced into the anterior $75 \%$ of the vertebral body.
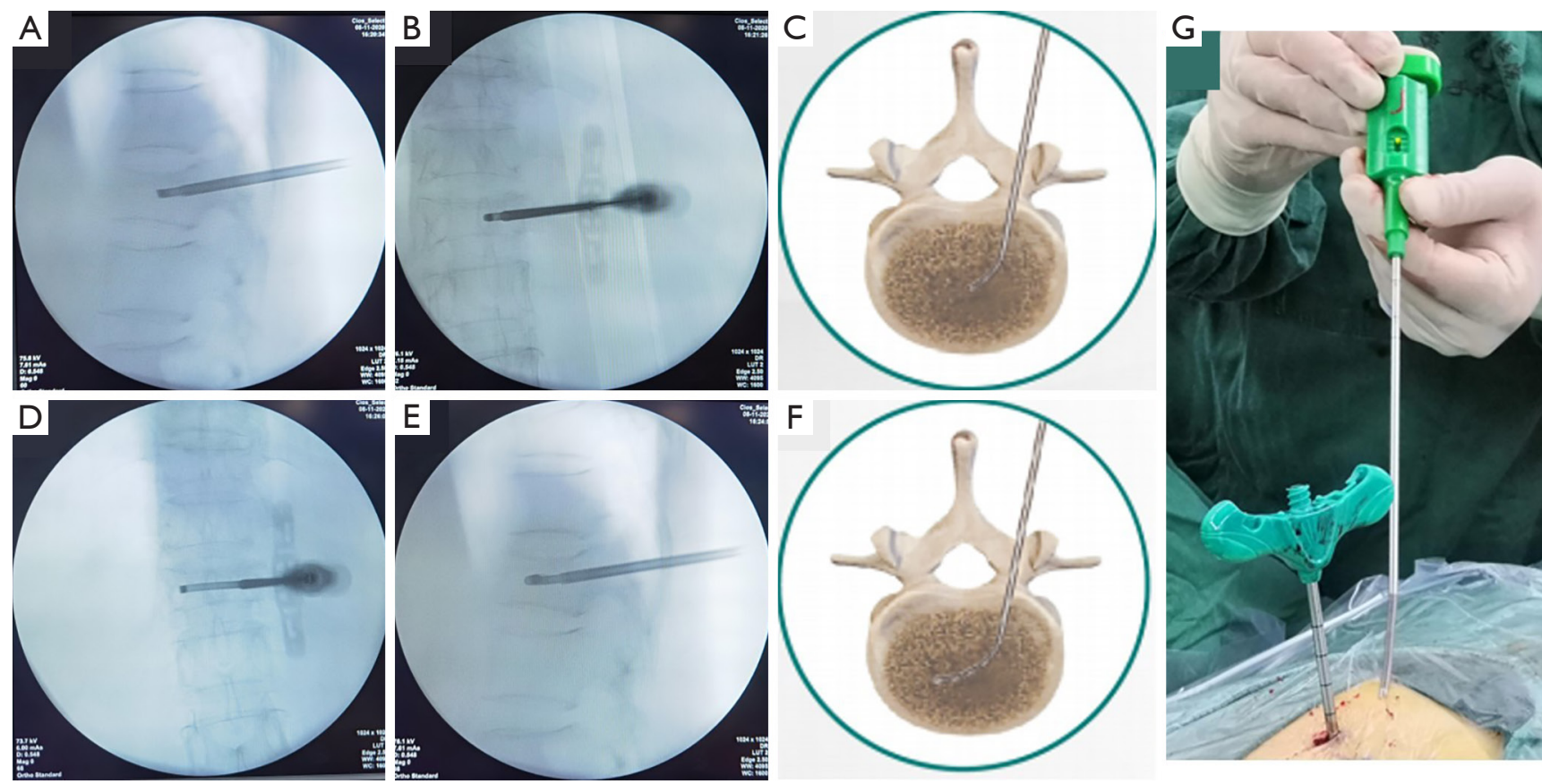

Figure 5 Steerable percutaneous kyphoplasty (S-PKP) surgical procedure in case one: (A,B,C) Steerable curved bone expander head bent to the middle area of the vertebral body; (D,E,F) steerable curved bone expander head further bent to the contralateral side of the vertebral body, establishing a curved cavity through the centerline; $(\mathrm{G})$ steerable curved bone expander.

the time to inject the bone cement were similar to the S-PKP procedure. Patients were also required to bed rest for the first 24 hours after surgery.

\section{Outcome measurement}

Clinical efficacy was evaluated using kyphotic Cobb's angle,
Oswestry disability index (ODI), visual analogue scale (VAS) score, injected cement volume, operation time and X-ray exposure times. Bone cement leakage and postoperative complications were also observed. It is defined that the primary endpoints included that the operative time and the times of intraoperative $\mathrm{X}$-ray fluoroscopy decreased, pain relieved, functional improved, no postoperative complications 


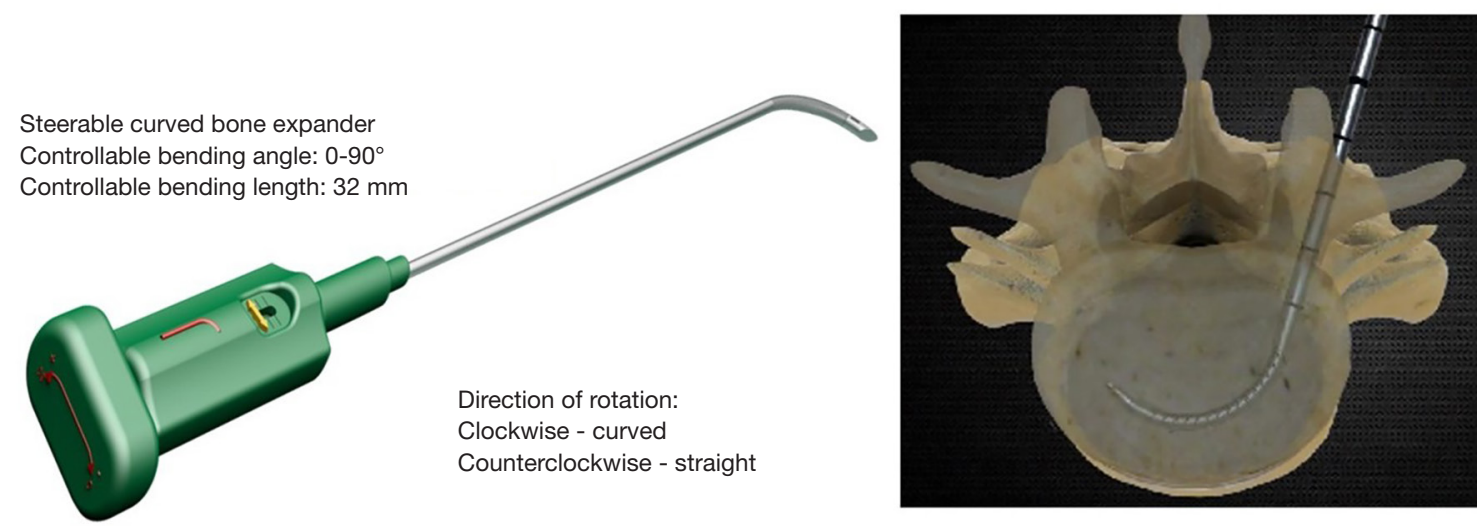

Figure 6 Steerable curved bone expander specifications and function.
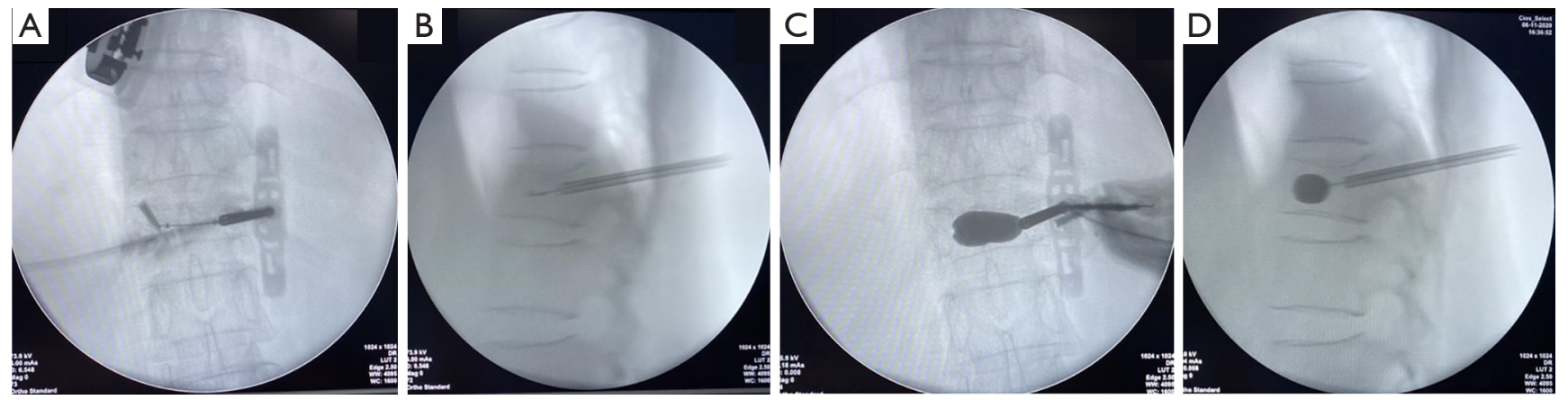

Figure 7 Steerable percutaneous kyphoplasty (S-PKP) surgical procedure in case one: $(\mathrm{A}, \mathrm{B})$ curved balloon tamp was inserted through the cannula and advanced into the anterior $75 \%$ of the vertebral body on the lateral view and in the middle area on the $\mathrm{AP}$ view; $(\mathrm{C})$ curved balloon tamp was inflated in the middle area of the vertebral body on the AP view; (D) curved balloon tamp was inflated in the anterior $75 \%$ of the vertebral body on the lateral view.

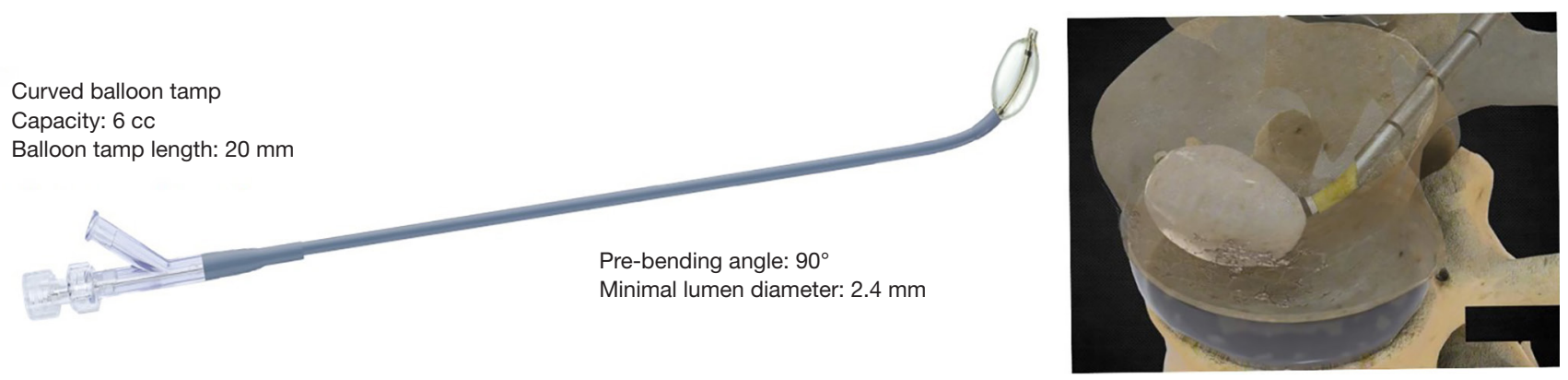

Figure 8 Curved balloon tamp specifications and function. 

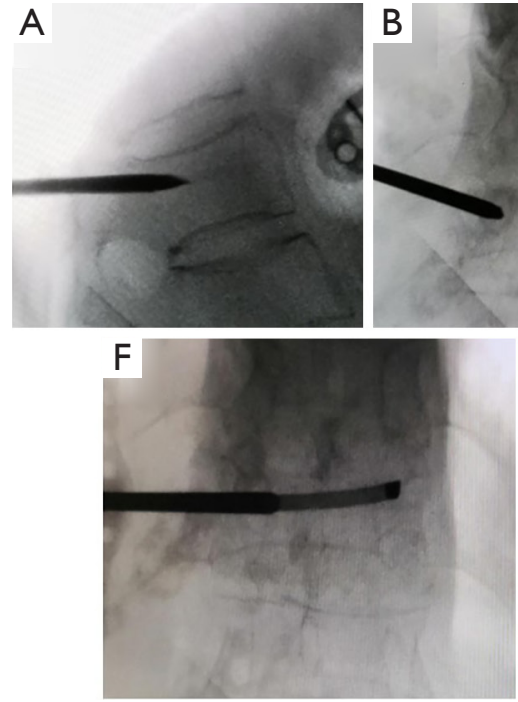
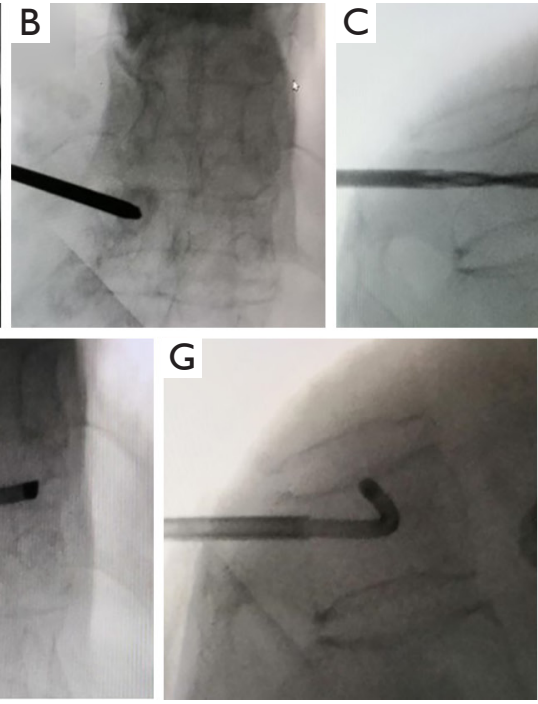
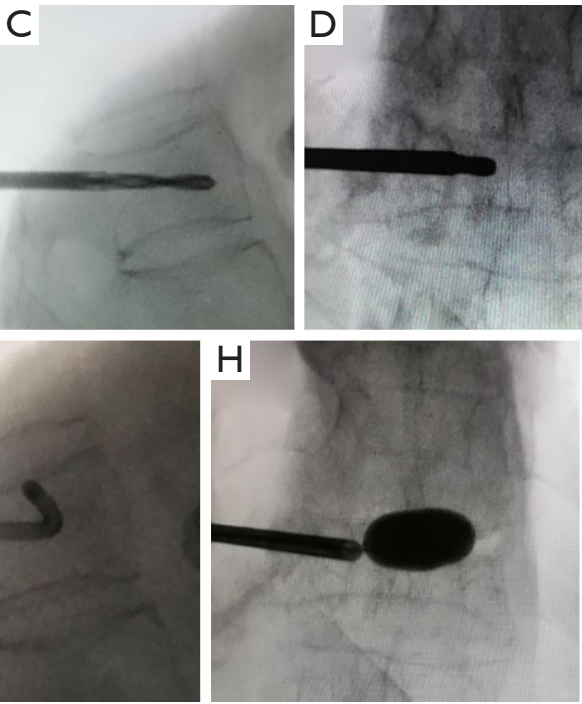
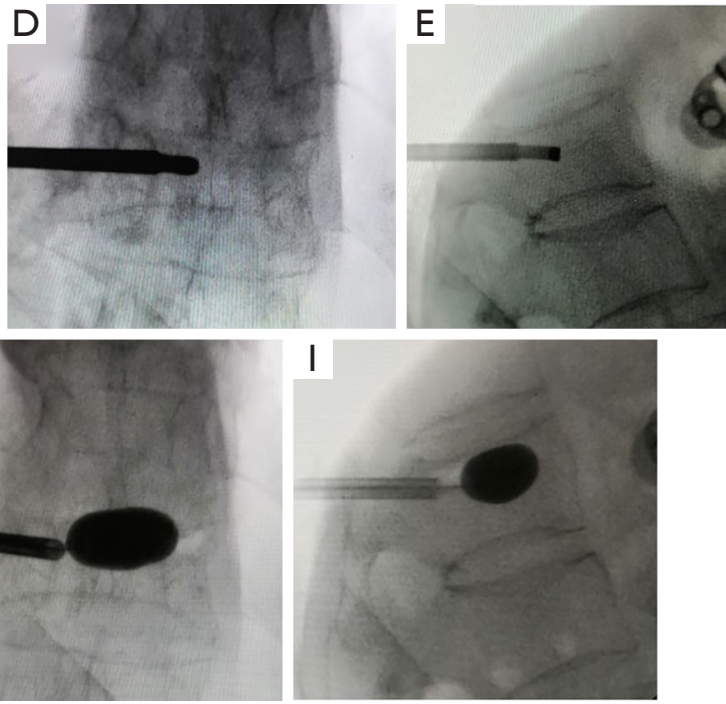

Figure 9 Steerable percutaneous kyphoplasty (S-PKP) procedure in case two. (A,B) Unilateral transpedicular approach; (C) bone drill advanced into the anterior $75 \%$ of the vertebral body; (D,E) steerable curved bone expander head bent to the middle area of the vertebral body, establishing a curved cavity through the centerline; (F,G) steerable curved bone expander head further bent to the contralateral side of the vertebral body; $(\mathrm{H}, \mathrm{I})$ curved balloon tamp was inserted through the cannula and inflated in the middle area of the vertebral body.
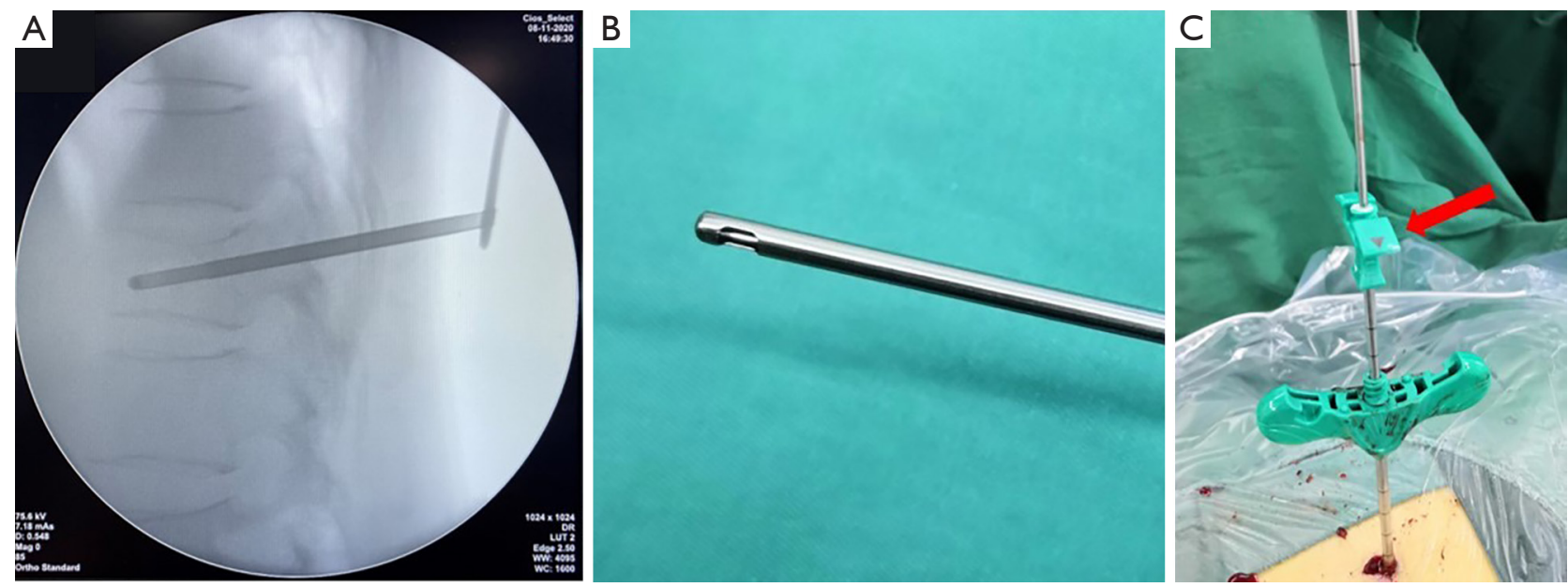

Figure 10 Application of Bone void filler device. (A) Bone void filler device inserted into the anterior $75 \%$ of the vertebral body; (B) bone void filler device with side hole; (C) arrow indicates the direction of cement diffusion.

happened. The secondary endpoints were the Cobb Angle going down. Patients were followed up once preoperatively, and at 1 day, 6 months, and 1 year postoperatively.

\section{Statistical analysis}

We estimated that a total of 150 patients would be needed to detect a difference between groups, with a two-tailed $\alpha$ of 0.05 and a $(1-\beta)$ of 0.80 . It should be noted that due to the new technology involved, we tried our best but failed to reach the expected number of cases within the prescribed time of the project, so more cooperative hospitals of centers should be included in subsequent experiments to ensure the sample size. SPSS 26.0 statistical software (version 26.0; SPSS, Inc., Chicago, IL, USA) was used to perform the difference analysis among different groups of variables. 

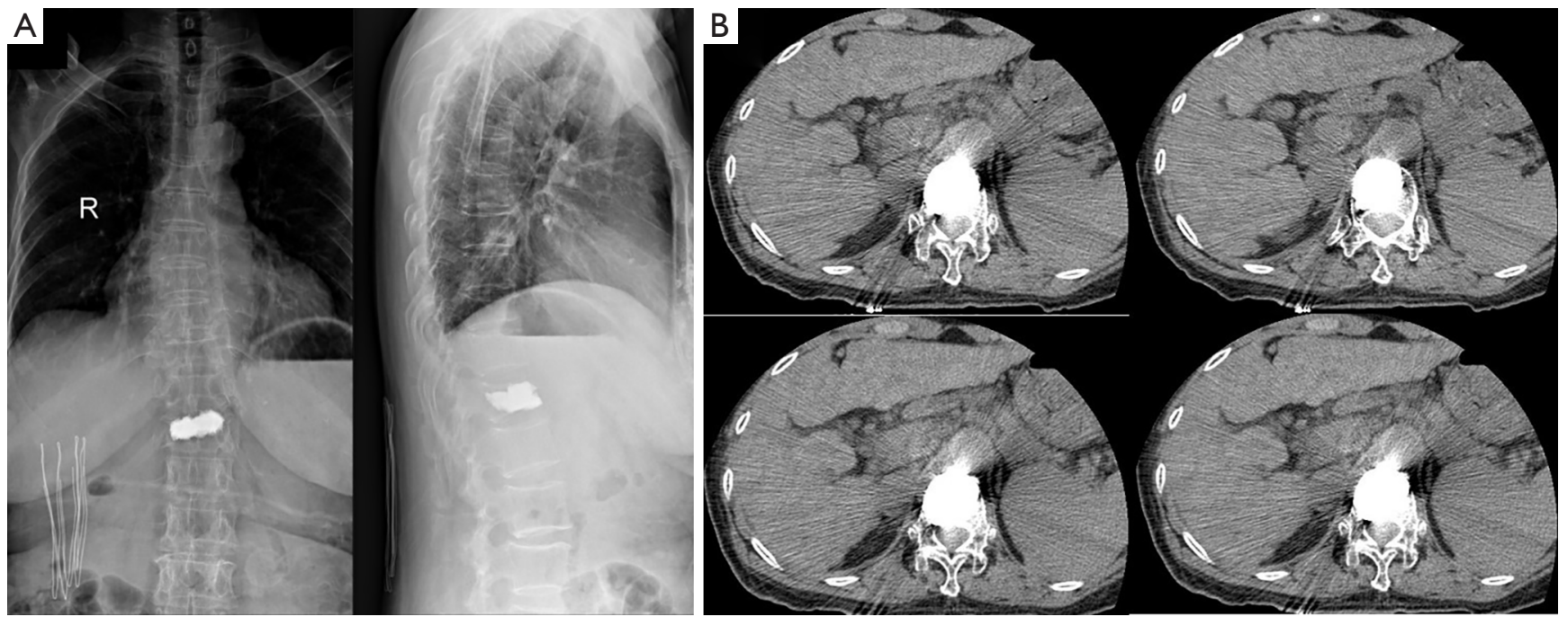

Figure 11 Postoperative X-ray and CT scan images in case one. (A) Postoperative X-ray images in the AP and lateral views revealed evenlydistributed bone cement and restoration of the vertebral body height; (B) postoperative CT images showing the distribution of bone cement after surgery.
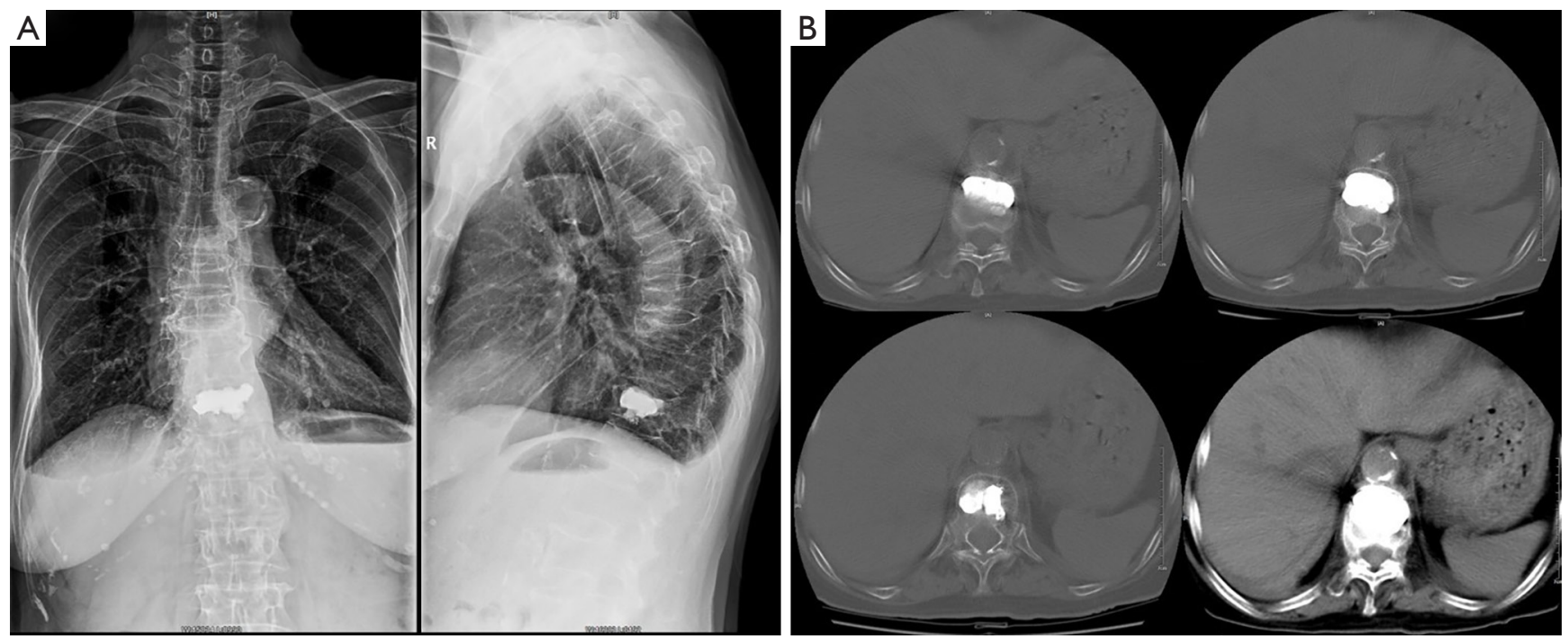

Figure 12 Postoperative X-ray and CT scan images in case two. (A) Postoperative X-ray images in the AP and lateral views revealed evenlydistributed bone cement and restoration of the vertebral body height. (B) Postoperative CT images showing the distribution of bone cement after surgery.

Enumeration data was compared by the chi-square test and expressed in the form of $\mathrm{n}(\%)$. The independent sample $\mathrm{T}$ test method was used to compare the measurement data between the two groups. Two-way ANOVA (Analysis of Variance)method was used to compare the measurement data between the two groups at different time points. The Least-Significant Difference (LSD) method was used to carry out pairwise comparison of the post-hoc test, which was expressed by $\bar{x} \pm \mathrm{s}$. A P value $<0.05$ indicated that the difference was statistically significant.

\section{Results}

From March 2019 to January 2020, patients from multi- 


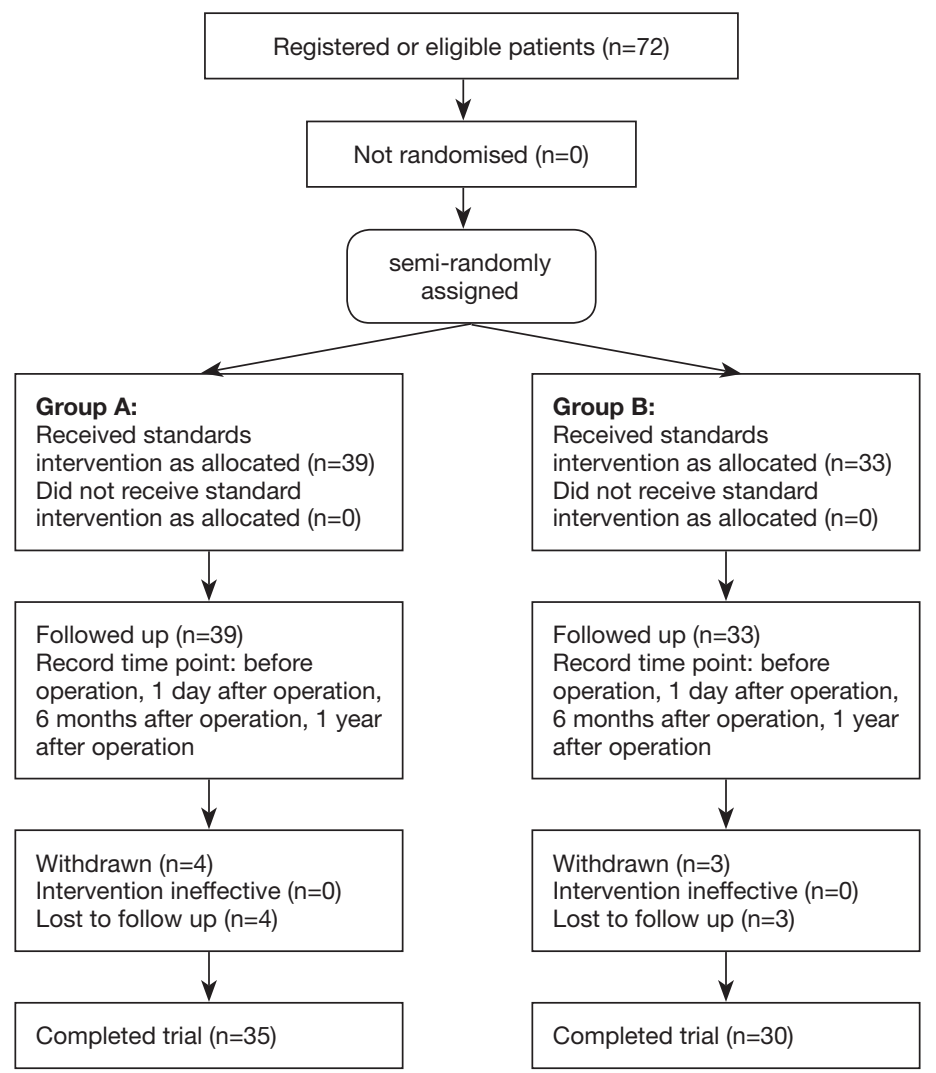

Figure 13 Flow diagram concerning participant enrolment.

center hospitals in Yunnan and Guizhou were enrolled in this study. Patients were followed up once preoperatively, and at 1 day, 6 months, and 1 year postoperatively.

\section{Comparative analysis of basic patient data between the two groups}

Secondary outcomes: there were 72 patients included in this study, including 39 patients in the PKP group and 33 patients in the S-PKP group (Figures 13). Surgery was performed successfully in all cases, and there were no cases of cement leakage or postoperative complications. The average age of patients in the PKP group was $74.79 \pm 6.61$ years, and the average age of patients in the S-PKP group was $77.06 \pm 6.63$ years. The mean BMD T value in the PKP group was $-3.45 \pm 0.50$, and the mean $\mathrm{BMD} T$ value in the S-PKP group was $-3.32 \pm 1.30$. There were no significant differences in gender, age, and $\mathrm{BMD} \mathrm{T}$ value between the two groups $(\mathrm{P}>0.05)$, and they were comparable (Table 1$)$.

\section{Comparative analysis of the surgical conditions between the two groups}

Primary outcomes: the operation time and radiation frequency of patients in the PKP group were significantly higher than those of the S-PKP group. The operation time was $51.59 \pm 9.14 \mathrm{~min}$ in the PKP group and $30.76 \pm 4.82 \mathrm{~min}$ in the S-PKP group. The frequency of intraoperative radiation was $105.9 \pm 31.93$ times in the $\mathrm{PKP}$ group and $47.42 \pm 11.88$ times in the S-PKP group. Intraoperative bone cement injection was approximately $5.25 \pm 1.37$ and $5.32 \pm 1.29 \mathrm{~mL}$ in the PKP and S-PKP groups, respectively (Table 2).

The results showed that S-PKP had advantages in reducing fluoroscopy times and shortening the operation time, and these differences were statistically significant $(\mathrm{P}<0.05)$. However, there was no notable difference in the amount of bone cement injection between the two groups $(\mathrm{P}>0.05)$. 
Table 1 Comparison of the basic data between the two groups

\begin{tabular}{|c|c|c|c|c|}
\hline Group & \multicolumn{2}{|c|}{ Gender } & Age (years) & BMD T value \\
\hline PKP Group (n=39) & $11(28.2)$ & $28(71.8)$ & $74.79 \pm 6.61$ & $-3.45 \pm 0.50$ \\
\hline S-PKP Group ( $n=33)$ & $9(27.3)$ & $24(72.7)$ & $77.06 \pm 6.63$ & $-3.32 \pm 1.30$ \\
\hline$\chi^{2} / \mathrm{t}$ & \multicolumn{2}{|c|}{0.008} & -1.447 & -0.580 \\
\hline
\end{tabular}

Table 2 Comparison results of operative conditions of patients between the two groups

\begin{tabular}{lccc}
\hline Group & Operation time $(\mathrm{min})$ & Bone cement injection amount $(\mathrm{mL})$ & Intraoperative radiation times (times) \\
\hline PKP Group $(\mathrm{n}=39)$ & $51.59 \pm 9.14$ & $5.25 \pm 1.37$ & $105.9 \pm 31.93$ \\
S-PKP Group $(\mathrm{n}=33)$ & $30.76 \pm 4.82$ & $5.32 \pm 1.29$ & $47.42 \pm 11.88$ \\
$\mathrm{t}$ & 12.346 & -0.248 & 10.602 \\
$\mathrm{P}$ & $<0.001$ & 0.805 & $<0.001$ \\
\hline
\end{tabular}

Table 3 Comparison of VAS scores between the two groups $(\bar{x} \pm \mathrm{s})$

\begin{tabular}{|c|c|c|c|c|c|c|}
\hline Group & \multicolumn{4}{|c|}{ VAS scores } & $\mathrm{F}$ & $P$ \\
\hline PKP Group & $7.57 \pm 0.92 c$ & $2.23 \pm 0.73 b$ & $1.2 \pm 0.76 \mathrm{a}$ & $1.14 \pm 0.49 a$ & 358.507 & $<0.001$ \\
\hline S-PKP Group & $7.7 \pm 1.02 c$ & $2.07 \pm 0.78 b$ & $1.2 \pm 0.66 \mathrm{a}$ & $1.2 \pm 0.66 \mathrm{a}$ & 314.614 & $<0.001$ \\
\hline $\mathrm{F}$ & 0.286 & 0.740 & 0.000 & 0.158 & & \\
\hline
\end{tabular}

Different lowercase letters indicate that the differences within the group were statistically significant $(\mathrm{P}<0.05)$; the same below.

\section{Comparative analysis of the VAS score and ODI between the two groups}

Primary outcomes: there were no significant differences in the VAS score and ODI between the two groups preoperatively $(\mathrm{P}>0.05)$. However, at the end of the study, these two scores were markedly reduced in both groups $(\mathrm{P}<0.05)$. Specifically, the preoperative VAS score of patients in the PKP group was $7.57 \pm 0.92$ points, which decreased to $2.23 \pm 0.73$ points at 1 day postoperatively, $1.2 \pm 0.76$ points at 6 months postoperatively, and $1.14 \pm 0.49$ points at 1 year postoperatively. In the S-PKP group, the preoperative VAS score was $7.7 \pm 1.02$ points, which decreased to $2.07 \pm 0.78$ at 1 day postoperatively, $1.2 \pm 0.66$ points at 6 months postoperatively, and $1.2 \pm 0.66$ points at 1 year postoperatively (Table 3 ).

In the PKP group, the preoperative ODI was
$87.62 \pm 7.26$ points, which decreased to $49.52 \pm 4.96$ points at 1 day postoperatively, $31.81 \pm 8.45$ points at 6 months postoperatively, and $24.63 \pm 7.14$ points at 1 year postoperatively. In the S-PKP group, the preoperative ODI was $86.67 \pm 6.04$ points, which decreased to $49.56 \pm 4.17$ points at 1 day postoperatively, $31.63 \pm 7.00$ points at 6 months postoperatively, and $24.89 \pm 5.26$ points at 1 year postoperatively (Table 4 ).

The results showed that the two surgical methods had the same effect in relieving pain and improving function of patients, and there were no significant differences $(\mathrm{P}>0.05)$.

\section{Comparative analysis of the Cobb angle of patients between the two groups}

Secondary outcomes: there was no significant difference in the Cobb angle between the two groups preoperatively 
Table 4 Comparison of ODI between the two groups $\left(\bar{x}_{ \pm s}\right)$

\begin{tabular}{lccccc}
\hline \multirow{2}{*}{ Group } & \multicolumn{4}{c}{ Oswestry disability index } & \multirow{2}{*}{$F$} \\
\cline { 2 - 4 } & Before surgery & 1 day after surgery & 6 months after surgery & 1 year after surgery & \\
\hline PKP Group & $87.62 \pm 7.26 \mathrm{~d}$ & $49.52 \pm 4.96 \mathrm{c}$ & $31.81 \pm 8.45 \mathrm{~b}$ & $24.63 \pm 7.14 \mathrm{a}$ & 455.564 \\
S-PKP Group & $86.67 \pm 6.04 \mathrm{~d}$ & $49.56 \pm 4.17 \mathrm{c}$ & $31.63 \pm 7.00 \mathrm{~b}$ & $24.89 \pm 5.26 \mathrm{a}$ & 373.580 \\
F & 0.324 & 0.001 & 0.001 & 0.026 & 0.001 \\
P & 0.571 & 0.978 & 0.927 & 0.872 \\
\hline
\end{tabular}

For the ODI and VAS scores in the last follow-up, a total of four patients in the PKP group were lost to follow-up, a total of three patients in the S-PKP group were lost to follow-up, and the shedding data were treated as missing values.

Table 5 Comparison of the Cobb angle between the two groups $\left({ }^{\circ}, \bar{x} \pm s\right)$

\begin{tabular}{|c|c|c|c|c|c|c|}
\hline Group & \multicolumn{4}{|c|}{ Cobb angle } & $\mathrm{F}$ & $\mathrm{P}$ \\
\hline PKP Group & $14.86 \pm 6.62 d$ & $8.18 \pm 4.71 a$ & $8.36 \pm 4.78 \mathrm{~b}$ & $8.73 \pm 4.83 c$ & 63.679 & $<0.001$ \\
\hline S-PKP Group & $13.98 \pm 5.28 d$ & $7.87 \pm 3.84 a$ & $8.11 \pm 3.87 b$ & $8.46 \pm 3.96 c$ & 52.840 & $<0.001$ \\
\hline $\mathrm{F}$ & 0.342 & 0.083 & 0.055 & 0.059 & & \\
\hline
\end{tabular}

$(\mathrm{P}>0.05)$, however the Cobb angle was improved considerably after surgery $(\mathrm{P}<0.05)$. In the PKP group, the Cobb angle was $14.86^{\circ} \pm 6.62^{\circ}$ preoperatively, $8.18^{\circ} \pm 4.71^{\circ}$ at 1 day postoperatively, $8.36^{\circ} \pm 4.78^{\circ}$ at 6 months postoperatively, and $8.73^{\circ} \pm 4.83^{\circ}$ at 1 year postoperatively. In the S-PKP group, the Cobb angle was $13.98^{\circ} \pm 5.28^{\circ}$ preoperatively, $7.87^{\circ} \pm 3.84^{\circ}$ at 1 day postoperatively, $8.11^{\circ} \pm 3.87^{\circ}$ at 6 months postoperatively, and $8.46^{\circ} \pm 3.96^{\circ}$ at 1 year postoperatively (Table 5 ).

The two surgical methods had the same effect in improving the Cobb angle, and there was no significant difference $(\mathrm{P}>0.05)$. However, the Cobb angle increased as the follow-up time was extended, which was considered to be related to the osteoporosis of patients themselves.

\section{Discussion}

\section{Advantages and disadvantages of unilateral and bilateral PKP}

In general, PKP is used to immediately relieve pain, as well as to improve the motor function and quality of life of OVCF patients. However, the radiation exposure, cement distribution, and vertebral stress concentration of two different approaches (unilateral/bilateral) PKP remains problematic. Chen et al. (11) showed that when bone cement was concentrated on one side of the vertebral body, the stress of the vertebral body would be unbalanced. However, when cement crosses the midline of vertebral body increase stress comparatively and biomechanical balance is thus achieved. Also, Lin et al. (12) reported that despite the advantages of unilateral puncture, the risk of arteriovenous injury and cement leakage during puncture is significantly increased by increasing the volume of cement injection in order to distribute cement evenly within the vertebral body. In a recent retrospective study, Lee et al. (13) pointed out that as a level 3 evidence and grade B recommendation, unipedicular kyphoplasty requires lower amounts of cement than bipedicular kyphoplasty and is as effective as bipedicular kyphoplasty in terms of radiological and clinical outcomes. Furthermore, Cui et al. (14) reported that unilateral PKP is superior to bilateral PKP in shortening operation time and bone cement leakage.

So, unilateral PKP requires a long learning curve and has an increased risk of postoperative complications. Bilateral PKP increases the radiation exposure and operation time. We believe that the novel S-PKP technology is one of the numerous possible solutions. 


\section{Key results analysis}

In this study, surgery was performed successfully in all cases, and there were no cases of cement leakage or postoperative complications. Both the PKP and S-PKP groups exhibited an ability to significantly decrease the total VAS scores, ODI, and Cobb angle; SPKP could achieve the same clinical effect as PKP. The VAS score in the PKP group decreased from $7.57 \pm 0.92$ preoperatively to $1.14 \pm 0.49$ at the last follow-up, and decreased from $7.7 \pm 1.02$ preoperatively to $1.2 \pm 0.66$ at the last follow-up in the S-PKP group. The ODI in the PKP group decreased from $87.62 \pm 7.26$ preoperatively to $24.63 \pm 7.14$ at the last follow-up, and decreased from $86.67 \pm 6.04$ preoperatively to $24.89 \pm 5.26$ at the last follow-up in the S-PKP group. The Cobb angle in the PKP group decreased from $14.86^{\circ} \pm 6.62^{\circ}$ preoperatively to $8.73^{\circ} \pm 4.83^{\circ}$ at the last follow-up, and decreased from $13.98^{\circ} \pm 5.28^{\circ}$ preoperatively to $8.46^{\circ} \pm 3.96^{\circ}$ at the last follow-up in the S-PKP group. In terms of shortening the operation time and reducing the frequency of radiation, S-PKP group was superior to the PKP group. The mean operation time of the S-PKP group was $30.76 \pm 4.82$ minutes, and was $51.59 \pm 9.14$ minutes in the PKP group. The average number of intraoperative C-arm fluoroscopy in the S-PKP group was $47.42 \pm 11.88$; and was $105.9 \pm 31.93$ in the PKP group. Thus, novel S-PKP technology can accommodate the advantages of bilateral and unilateral PKP in the treatment of OVCFs.

\section{The innovation and advantages of S-PKP}

The S-PKP procedure's use of the unilateral transpedicular approach can achieve a bilateral puncture effect, improve puncture efficiency and success rate, and reduce the operation time and radiation exposure. The steerable curved bone expander can establish a curved cavity cross the centerline, and the bone cement is evenly diffused. Center injection of bone cement enhances vertebral stability, and decreases stress concentration and bone cement leakage.

\section{Our surgical experiences}

Based on our surgical experiences, the standard position of steerable curved bone expander is as follows: the head of bone expander should be projected at the midpoint of the contralateral pedicle on the AP view, and should be projected on the center or anterior middle third of the vertebral body on the lateral view. Also, the standard position of the curved balloon tamp is in the middle of the vertebral body (between the bilateral pedicles) on the $\mathrm{AP}$ view and at the center or anterior middle third of the vertebral body on the lateral view.

When using the balloon tamp, attention should be paid to observe the location and volume of balloon expansion; if one balloon expansion fails to achieve vertebral reduction, multiple balloon dilations can be performed. The balloon tamp expansion pressure should not exceed 300PSI, and expansion volume should not exceed 6cc. The balloon stops expanding when it reaches the endplate or one side of the vertebral cortex.

\section{Conclusions}

In conclusion, our findings indicate that novel S-PKP can reduce the operation time and radiation exposure, and improve the postoperative quality of life of OVCF patients. The limitations of this study include the small sample size, and the subjectivity of a satisfactory postural correction. Therefore, randomized controlled studies involving larger sample sizes are still needed in the future.

\section{Acknowledgments}

Funding: This study was supported by Yunnan Province Clinical Center for Bone and Joint Diseases Programme (ZX2019-03-04).

\section{Footnote}

Reporting Checklist: The authors have completed the CONSORT reporting checklist. Available at https://dx.doi. org/10.21037/atm-21-1880

Trial Protocol: Available at https://dx.doi.org/10.21037/atm21-1880

Data Sharing Statement: Available at https://dx.doi. org/10.21037/atm-21-1880

Conflicts of Interest: All authors have completed the ICMJE uniform disclosure form (available at https://dx.doi. org/10.21037/atm-21-1880). The authors have no conflicts of interest to declare.

Ethical Statement: The authors are accountable for all aspects of the work in ensuring that questions related 
to the accuracy or integrity of any part of the work are appropriately investigated and resolved. This study was approved by the Ethics Committee of Yunnan Hospital of Traditional Chinese Medicine/ Medical Ethics Committee of the First Affiliated Hospital of Yunnan University of Traditional Chinese Medicine (2021-034). All procedures performed in this study involving human participants were in accordance with the Declaration of Helsinki (as revised in 2013). Informed consent was obtained from all patients.

Open Access Statement: This is an Open Access article distributed in accordance with the Creative Commons Attribution-NonCommercial-NoDerivs 4.0 International License (CC BY-NC-ND 4.0), which permits the noncommercial replication and distribution of the article with the strict proviso that no changes or edits are made and the original work is properly cited (including links to both the formal publication through the relevant DOI and the license). See: https://creativecommons.org/licenses/by-nc$\mathrm{nd} / 4.0 \%$.

\section{References}

1. Buchbinder R, Johnston RV, Rischin KJ, et al. Percutaneous vertebroplasty for osteoporotic vertebral compression fracture. Cochrane Database Syst Rev 2018;4:CD006349.

2. Galibert P, Deramongd H, Rosat P, et al. Preliminary note on the treatment of vertebral anginoma by percutaneous acrylic vertebroplasty. Neurochirurgie 1987;33:166-8.

3. Yan D, Duan L, Li J, et al. Comparative Study of Percutaneous Vertebroplasty and Kyphoplasty in the Treatment of Osteoporotic Vertebral Compression Fractures. Arch Orthop Trauma Surg 2011;131:645-50.

4. Wang H, Sribastav SS, Ye F, et al. Comparison of Percutaneous Vertebroplasty and Balloon Kyphoplasty for the Treatment of Single Level Vertebral Compression Fractures: A Meta-analysis of the Literature. Pain Physician 2015;18:209-22.

5. Huang S. Therapeutic Effect of Percutaneous Kyphoplasty combined with Anti-Osteoporosis Drug on Postmenopausal Women With Osteoporotic Vertebral Compression Fracture and Analysis of Postoperative Bone Cement Leakage Risk Factors: A Retrospective Cohort Study. J Orthop Surg Res 2019;14:452.

6. Lieberman IH, Dudeney S, Reinhardt MK, et al. Initial outcome and efficacy of "kyphoplasty" in the treatment of painful osteoporotic vertebral compression fractures. Spine (Phila Pa 1976) 2001;26:1631-8.

7. Garfin SR, Yuan HA, Reiley MA. New technologies in spine: kyphoplasty and vertebroplasty for the treatment of painful osteoporotic compression fractures. Spine 2001;26:1511-5.

8. Yilmaz A, Çakir M, Yücetaş CŞ, et al. Percutaneous Kyphoplasty: Is Bilateral Approach Necessary? Spine (Phila Pa 1976) 2018;43:977-983.

9. Feng H, Huang $\mathrm{P}$, Zhang X, et al. Unilateral versus bilateral percutaneous kyphoplasty for osteoporotic vertebral compression fractures: A systematic review and meta-analysis of RCTs. J Orthop Res 2015;33:1713-23.

10. Tang J, Guo WC, Hu JF, et al. Unilateral and Bilateral Percutaneous Kyphoplasty for Thoracolumbar Osteoporotic Compression Fractures. J Coll Physicians Surg Pak 2019;29:946-50.

11. Chen B, Li Y, Deng H, et al. Comparison of unipedicular and bipedicular kyphoplasty on the stiffness and biomechanical balance of compression fractured vertebrae. Eur Spine J 2011;20:1272-80.

12. Lin D, Hao J, Li L et al. Effect of Bone Cement Volume Fraction on Adjacent Vertebral Fractures After Unilateral Percutaneous Kyphoplasty. Clin Spine Surg 2017;30:E270-5.

13. Lee CH, Kim HJ, Lee MK, et al. Comparison of efficacies of unipedicular kyphoplasty and bipedicular kyphoplasty for treatment of single-level osteoporotic vertebral compression fractures: A STROBEcompliant retrospective study. Medicine (Baltimore) 2020;99:e22046.

14. Cui Z, Tian Y, Feng H, et al. Unilateral Versus Bilateral Balloon Kyphoplasty for Osteoporotic Vertebral Compression Fractures: A Systematic Review of Overlapping Meta-analyses. Pain Physician 2019;22:15-28.

(English Language Editor: A. Kassem)

Cite this article as: Li J, Yuan X, Li F, Ding Y, Ma G, Song C, Chen X, Wang E, Cui J, Kong Q, Huang Y, Song E. A randomized trial comparing the clinical efficacy and safety of a novel steerable percutaneous kyphoplasty with traditional PKP in osteoporotic vertebral fractures. Ann Transl Med 2021;9(12):1024. doi: 10.21037/atm-21-1880 